\title{
Wide angle digital holographic interferometry with real-time optical reconstruction
}

\author{
Malgorzata Kujawinska, ${ }^{* 1}$ Grzegorz Finke, ${ }^{1}$ Piotr Garbat, ${ }^{1}$ Claas Faldorf ${ }^{2}$, Bryan M. Hennelly ${ }^{3}$ \\ ${ }^{1}$ Institute of Micromechanics and Photonics, Warsaw University of Technology, \\ 8 Sw. A.Boboli St., Pl-02-525 Warsaw, Poland \\ ${ }^{2}$ BIAS - Bremer Institut für angewandte Strahltechnik GmbH Klagenfurter Straße 2, D - 28359 Bremen, \\ Germany \\ ${ }^{3}$ Department of Computer Science, National University of Ireland, Maynooth, Co. Kildare, Ireland
}

Received June 24, 2012; accepted June 29, 2012; published June 30, 2012

\begin{abstract}
In the paper we will discuss the wide angle capture and display systems applied for digital holographic interferometry (DHI) The static capture system is based on a single camera and an object located at a rotational stage. The holograms are captured sequentially in time during object loading and the phases at a hologram plane are calculated by phase shifting method. The dynamic capture system employs several cameras located around an object and simultaneous capture of several holograms is performed with the frequency of impulse laser applied. For both DHI capture systems the holographic display configuration is proposed. Multiple spatial light modulators aligned in circular configuration are put to work together. In the paper the main requirements for optical reconstruction of resultant interferograms originated from both capture systems are discussed. The results of experiments are presented and analyzed.
\end{abstract}

Holographic interferometry (HI) is used to observe changes of an object's phase during its external or internal loading [1]. In order to see these changes a sequence of holograms has to be registered. In classical double exposure HI two holograms are register on the same hologram. During reconstruction of this hologram an interference pattern which visualize an object phase difference is obtained. After interferogram analysis the quantitative phase data are obtained. Conventional HI requires inconvenient chemical processing of holographic plates. The progress in high resolution $\mathrm{CCD} / \mathrm{CMOS}$ detectors enabled wide application of digital holography (DH) and DH interferometry (DHI). DH and DHI brought different, simplified and digital way of holograms recording and their numerical reconstruction [2]. The next step to mimics classical $\mathrm{HI}$ is to use electronically addressed spatial light modulators with digital holographic data and reconstruct optically digital holograms formed at SLMs [3]. In such case the procedure of registration and data processing may be realized in two ways:

*E-mail: m.kujawinska@mchtr.pw.edu.pl
- direct holograms manipulation and display (off-axis holograms have to be recorded and displayed),

- calculation of phases in a hologram plane and manipulation of the phases (for a phase calculation inline phase shifting holograms [1] are usually applied, which requires capturing at least 3 PS).

The first case is usually used for monitoring of dynamic changes in an object, while the second one delivers good quality interferograms for static events.

The main disadvantage of DH and DHI is a limited bandwidth spatial product of array detectors and SLMs which resolution is well below this of conventional silver halide holographic material. To overcome at least partly this problem two approaches are proposed:

- DHI based on in-line holography and hologram display at SLM (in this case full detector and SLM spatial bandwidth can be used),

- using multiple CCDs and multiple SLMs to gather and display information from much wider angular field of view.

Below both approaches are discussed and implemented.

Experimental capture and reconstruction setup:

The first DH capture setup is shown in Fig. 1. It allows to capture a sequence of in-line, phase shifted holograms for all stages of an object which is loaded in a step-like mode [3]. The object can be rotated and the load sequence can be repeated in order to see the behavior of the object in bigger angular field of view (from different directions). The phase shifting is realized by a mirror with PZT pusher placed in the reference beam. The CCD camera with 1920x1080pixels with pixel pitch $7.4 \mu \mathrm{m}$ is here applied. 


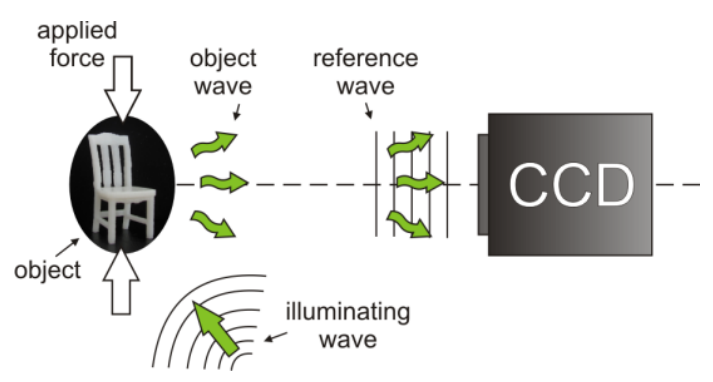

Fig. 1. The scheme for registration of a sequence of in-line digital holograms of an object under load.

DHs registration system allowing to capture an object within extended angular field of view is presented in Fig. 2. The six sensors are arranged in a circular arc respecting a point-shaped object position [4]. Each CCD (2056x2452 pixels with pixel pitch $3.45 \mu \mathrm{m})$ covers an angle of $1.6^{\circ} \pm 0.1^{\circ}$, however the cameras (due to their physical size) have the angular separation of $15^{\circ} \pm 1^{\circ}$. i.e. there are significant gaps between the cameras and the object field captured. The consequence of this is that we are not able to stitch optically full object field during reconstruction but we will reconstruct separately in space object waves from different perspectives. Providing continuous wavefield (at the observer's resolution level) is important from imaging (3D display) point of view [4], but in metrology we often it is sufficient that we are able to monitor simultaneously in time an object from different perspectives (separate interferograms).

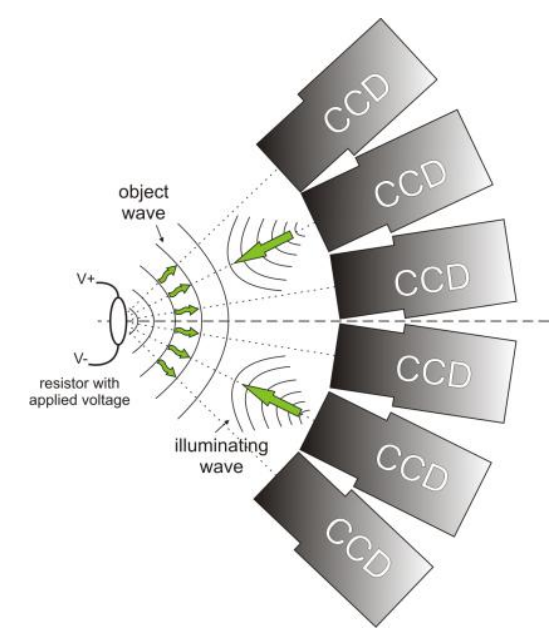

Fig. 2. The scheme for registration of a sequence of set of 6 holograms providing information for wide angle DHI.

Capturing an extended field of view digital hologram of a dynamic scene requires short exposure times. Here we had applied a pulsed laser which provides pulses of $6 \mathrm{~ns}$ at a wavelength of $532 \mathrm{~nm}$. The laser beam has been expanded and collimated before being split into one or two object illumination and six reference waves. Each of the reference waves impinges slightly off-axis on each of the six CCDs and allow to capture digital off-axis Fresnel holograms [1]. The synchronised capture of six single DHs from different perspectives is ensured by a trigger device, which outputs a periodical signal to all six sensors by activating the measurement. Since the maximum pulse rate of the pulsed laser is limited to $10 \mathrm{~Hz}$, the capturing rate is limited to the same value, so the system allows for real time, remote, wide object angle digital holographic interferometry which may compare a state of an object with maximum frequency $5 \mathrm{~Hz}$.

In order to reconstruct optically digital holograms (or their phases) representing different perspectives of an object, we have built a display system composed of six Liquid Crystal on Silicon (LCoS) SLMs in circular configuration (Fig.3). The display is an assembly of two modules: illumination and reconstruction [3, 4]. The task of the first one is to illuminate SLMs (1920x1080 pixels with pixel size $8 \mu \mathrm{m}$ ) with set of parallel, homogeneous, coherent and linearly polarized beams. The display arrangement with parallel illuminating beams is different to the capture one where CCDs are illuminated from normal direction. This is one of the reason why we cannot use a simple transmission of a hologram into LCoS but we calculate and modify by a tilt procedure a phase at a hologram plane. SLMs are address with this phase. In the reconstruction module there are six SLMs arranged on the circle of radius $\mathrm{R}$. The reconstruction distance and angular distribution of SLMs depend on the capture configuration and/or the data processing module as marked schematically in Fig. 3 by different positions of the images of reconstructed objects.

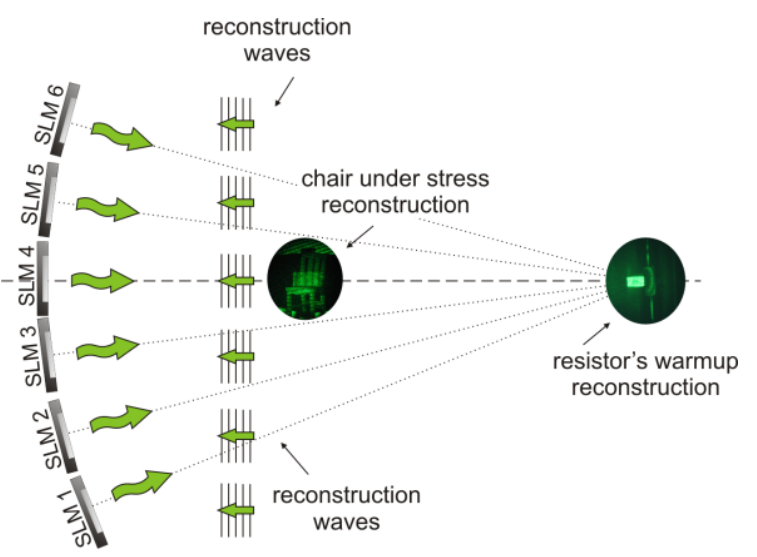

Fig. 3. The scheme of 3D DH/DHI display based on 6 LCoS SLMs. The images of chair and resistor indicate different localization of images reconstructed from the first and second capture system, respectively.

\section{Experimental results:}

The DHI experiments had been performed in two stages. At first a sequence of phase-shifted Fresnel in-line holograms of unloaded and sequentially (step-like) tensile loaded small model of a plastic chair $(30 \mathrm{~mm}$ height, 
located 436mm from CCD) were captured in the system with a single camera. The sequential phases are calculated from phase shifted holograms. In this procedure all unwanted orders from hologram reconstruction are eliminated. It is well shown in the numerically obtained interference fringes (Fig. 4a). In the case of optical reconstruction the sequential phases values are addressed at LCOS so that both wavefronts (reference and actual state) are generated and interfere showing the phase difference in the form of an interferogram. The result is shown in Fig.4b. In the centre we can see a weak image of a zero order beam, which is due to reflections from LCoS. The quality of fringes in both cases is very good. Similar results will be obtained if we rotate the object and for the same sequence of loads capture holograms. In this way we can get several series of object displacement maps covering 360deg view of a static object loaded with well controlled step like forces.
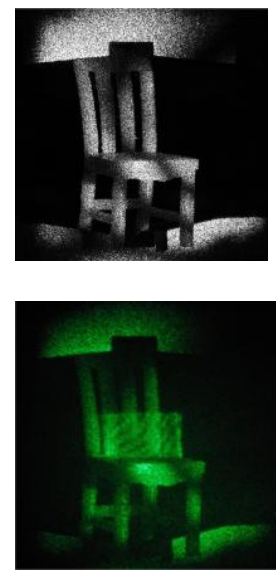

Fig. 4. The resultant,

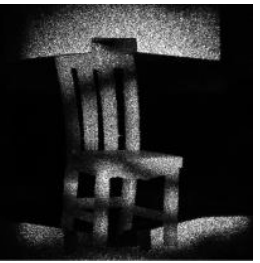

a)

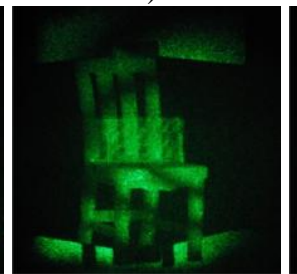
increasing tensile force: a) a set of numerically reconstructed interferograms and $b$ ) the relevant interferrograms reconstructed optically by LCOS SLM.

Next experiment was performed for the multi camera capture system. An object was a resistor $(30 \mathrm{~mm}$ with wires, located 260mm from CCDs) continuously loaded thermally by passing current what induced out-of plane displacements. In this case no phase shifting procedure was possible and the phases have to be obtained from a single hologram. Therefore we captured off-axis holograms and the phases were calculated by Fourier transform method [1]. During optical reconstruction we had observed the resistor from 6 perspectives in wide angle (the interferograms were visualized at a diffuser), which allows in principle to monitor the resistor behavior in 90deg angular field of view (Fig. 5). Note that in this case the zero diffraction order is not removed and it disturb the central part of the image. There are numerical methods to remove unwanted zero and twin orders [5], however they are usually used for imaging purposes only as they may disturb the phases of an object which in the case of DHI is highly undesired. Anyhow the interferograms obtained from pairs of holograms captured from different object perspectives carry information about an object displacements.
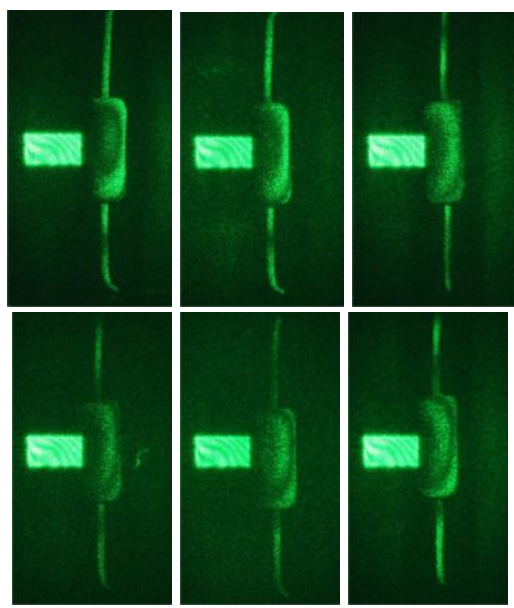

Fig. 5. The six interferograms obtained simultaneously in 3D DH display during the analysis of the thermally loaded resistor.

In the paper we had shown that coupling the multi camera capture system and multi LCoS SLMs DH reconstruction system allows to monitor object displacements in wide angular field of view. However the reconstructed wavefronts are not stitched coherently. The main reason for this is that the captured object field has significant gaps, so the wavefronts cannot form a continuous interferogram, but they can be reconstructed separately. The future works will focus on development of capture and reconstruction DHI systems which provide true (coherently stitched) wide angle object field monitoring capabilities.

The research leading to these results has received funding from the EU $7^{\text {th }} \mathrm{FP}$ within the project Real3D under agreement 216105 and from National Scientific Centre within the project HoloTrue3D under agreement 2011/02/A/ST7/00365.

\section{References}

[1] T. Kreis, Handbook of holographi interferometry: Optical and digital methods (Wiley-VCH Verlag GmbH, 2005).

[2] A. Michałkiewicz, M. Kujawińska, K. Stasiewicz, Optoelectr. Rev. 16, 68 (2008).

[3] T. Kozacki, M. Kujawinska, G. Finke, B. Hennely, N. Pandey, Appl. Opt. 52, 1771 (2012)

[4] M. Kujawinska, T. Kozacki, Holographic Television: Status and Future, in Optical Imaging and Metrology: Selected Topics, 55-93, (eds W. Osten and N. Reingand; Wiley-VCH Verlag GmbH, 2012).

[5] D.P. Kelly, et al., Int. J. Digital Multimedia Broadcasting , art. ID 759323 (2010). 